\title{
Los nombres de acción en algunos diccionarios del español
}

\author{
María Ángeles García García-Serrano \\ Universidad de Castilla-La Mancha
}

\section{INTRODUCCIÓN}

En los últimos diez años hemos asistido a la publicación creciente de diccionarios del español -DRAE (1992), DUE (1998), DEA (1999), $D G L E$ (1997), CLAVE (1996), etc.- en los cuales se han introducido todo tipo de innovaciones estéticas y tecnológicas siguiendo las más hábiles estrategias de marketing. Sin embargo, la renovación de las características físicas del libro no ha ido acompañada de la mejora en la calidad lexicográfica que requerían algunas de sus entradas, como es el caso de los miles de artículos destinados a los denominados nombres de acción.

Es cierto que hay siempre que contar con que los diccionarios resultan incompletos y no cabe en ellos la riqueza léxica que presenta la realidad lingüística. La causa principal y obvia de este defecto es el carácter selectivo de lo que pretende ser un manual. El contenido de un diccionario de uso ha de ceñirse a lo esencial, es decir, a un vocabulario de uso frecuente, contemporáneo y general y debe excluir lo que está en desuso o es de uso muy limitado en determinada región, capa social o campo temático. Otra razón -no menos importante, pero menos obviaes la separación entre la parte práctica de la lexicografía, con la elaboración de diccionarios, y la teoría lingüística. Y es justo aquí donde situamos la posición poco coherente de los diccionarios en la definición del proceso de formación de palabras que nos ocupa.

Como tenemos presente que los diccionarios no están destinados a los lingüistas sino al público en general, el objetivo de este artículo es 
aclarar ciertas cuestiones y problemas que presenta la descripción de los nombres de acción en los diccionarios actuales del español más que aportar una información teórica indiscriminada. Al mismo tiempo, no obstante, estaremos mostrando la forma en la que pueden relacionarse de manera efectiva los aspectos teóricos de la morfología con definiciones lexicográficas adecuadas, válidas y suficientes que puedan satisfacer tanto las necesidades de ese público en general como las del especialista.

El contenido de este artículo está organizado del siguiente modo: en primer lugar, presentamos la descripción que desarrollan los diccionarios de uso de los principales sufijos que participan en el proceso morfológico de la nominalización. A continuación, presentamos la forma en la que son descritos los propios sustantivos derivados. Efectuaremos, por tanto, una evaluación crítica de los procedimientos descriptivos a los que recurren los diccionarios actuales del español que ponga de manifiesto las limitaciones y la inconsistencia de la representación lexicográfica que reciben estos nombres deverbales, en particular, y de la descripción de este proceso derivativo, en general.

\section{LOS SUFIJOS NOMINALIZADORES EN LOS DICCIONARIOS ACTUALES DEL ESPAÑOL}

La formación de nombres deverbales de acción en español es un proceso morfológico altamente productivo por su regularidad, ya que está sometido a pocas restricciones y es semánticamente transparente, y por la disponibilidad del afijo (preferentemente -ción) para construir nuevas formaciones. La teoría gramatical ${ }^{1}$ viene definiendo este proceso como la formación de nombres a partir de verbos mediante la adjunción de un sufijo con una determinada forma (-ción, por ejemplo) y un contenido semántico ('acción de V'). Las investigaciones más recientes (cfr. Beard 1995, García 2002) consideran, sin embargo, que este proceso de sufijación transforma únicamente la categoría de la pieza léxica de ver-

1 Véase Santiago Lacuesta y Bustos Gisbert (1999), Fernández Ramírez (1986), Monge (1970), Pena (1976, 1980), entre otros. 
bo a nombre pero no añade ningún significado semántico adicional. El significado de la palabra derivada está determinado por las propiedades (semánticas, sintácticas y aspectuales) de la base de derivación. Esto es, si el proceso de nominalización tiene lugar sobre un verbo como construir, la palabra derivada denotará 'la acción de construir'. Si tomamos como base de derivación un verbo estativo como conocer, el sustantivo derivado denotará 'el estado de tener o estar en posesión de cierto saber'. Será, en cualquier caso, a partir de este significado inicial, y una vez incorporado al léxico, cuando el sustantivo podrá desarrollar nuevas significaciones a través de un desplazamiento metonímico. Así, es normal que los derivados que indican acciones puedan referirse también al resultado de las mismas (construcción, descubrimiento, alabanza, compra, etc.) y, tan sólo en ocasiones, pueden también desarrollar otros significados secundarios como son los valores agentivo (organización, asociación, dirección, etc.); instrumental (compensación, gratificación, embalaje, etc.); locativo (habitación, establecimiento, residencia, etc.); o temporal (floración, lactancia, regencia, etc.).

Los diccionarios antes mencionados formalizan esta información de manera similar. Otorgan al sufijo nominalizador el significado de 'acción y efecto', aunque para ello acudan a criterios diferentes: bien incorporan los sufijos en el cuerpo de la obra, ordenados alfabéticamente, bien se registran en una lista anexa al final del diccionario o bien no se incluyen. El Diccionario de la lengua española de la Real Academia (DRAE), el Diccionario general de la lengua española (DGLE) y el Diccionario de uso del español (DUE) incluyen el sufijo dentro de la macroestructura de la obra. Veamos sucintamente cómo son descritos en estos diccionarios los principales sufijos que forman nombres de acción.

La Academia define los principales sufijos de la siguiente forma:

(1) Definición de DRAE (1992)

- ción. (Del lat. -ť̈o, -ōnis.) suf. de sustantivos verbales que significa acción y efecto. Aparece en la forma -ción, no precedido de vocal, en ciertos sustantivos generalmente procedentes del latín: funCIÓN, lecCIÓN, producCIÓN. Los creados en español toman la forma -ación, si el verbo del que derivan es de la primera conjugación: grabACIÓN; -ición, si es de la tercera: embutICIÓN. Si el sustantivo deriva de un 
verbo de la segunda, toma otro sufijo. Además de su significado abstracto, -ción y sus variantes pueden denotar objeto, lugar, etc.: embarCACIÓN, fundICIÓN.

- $d a^{2}$.- suf. de sustantivos derivados de otros sustantivos o de verbos. $\mathrm{Si}$ el verbo es de la primera conjugación, toma la forma -ada; -ida si es de la segunda o de la tercera. [...] Los derivados de verbos suelen denotar acción y efecto: IlamADA, llegADA. A veces, -ada se combina con otros sufijos, como -ar: lumbrARADA, IlamARADA; y -arro: nubARRADA. La variante -ida forma sustantivos que generalmente significan acción y efecto: $\operatorname{acogIDA}$, acometIDA, partIDA, sacudIDA.

- dura. (Del lat. -tura). suf. de sustantivos verbales. Toma las formas -adura, -edura o -idura según que el verbo base sea de la primera, segunda o tercera conjugación. Significa acción y efecto: salpicADU$R A$, soldADURA, torcEDURA, mordEDURA, añadIDURA, hendIDURA; a veces denota el medio o instrumento de la acción: cerrADURA; o conjunto: arbolADURA.

- miento. (Del lat. -mentum). suf. de sustantivos verbales que suele significar acción y efecto. Toma la forma -amiento cuando el verbo base es de la primera conjugación: debilitAMIENTO, levantAMIENTO; -imiento, si es de la segunda o de la tercera: atrevIMIENTO, florecIMIENTO, aburrIMIENTO, sentIMIENTO.

Como vemos, en el $D R A E$ se explica la categoría de la base a la que el sufijo se adjunta y las variantes fonéticas del propio sufijo. En cambio, no se da información sobre el comportamiento sintáctico de la pieza léxica derivada y el propio significado del sufijo aparece muy confuso. Si nos fijamos en la entrada de -ción, vemos que en primer lugar se define como 'acción y efecto'. De lo que deduciremos erróneamente que toda palabra formada con dicho sufijo tendrá sistemáticamente ambas denotaciones. Al final de la entrada podemos leer "aparte de su significado abstracto [...] puede denotar objeto, lugar”. Ante esto, ¿el usuario debe interpretar que existe un proceso morfológico por el que se crean nombres abstractos con la denotación de acción y efecto (con el

2 En la última edición del diccionario académico se ha suprimido la entrada de este sufijo (véase DRAE-2001, s. v. -ada). 
problema de considerar todo efecto como abstracto) y otro proceso morfológico distinto por el que el sufijo crea nombres de objeto o de lugar? Lógicamente ésta es la interpretación que se extrae de la definición dada; sin embargo, no es una descripción teóricamente correcta de este proceso de formación de palabras.

En el Diccionario de uso del español (DUE) la información que se da de cada uno de los sufijos es semejante a la del diccionario de la Academia:

(2) Definiciones del DUE

-ción: Parte invariable del sufijo «-ación, -ición» con que se forman nombres de acción derivados de verbos: 'mediación, aparición, reparación'. También, nombres de función o cargo: 'inspección, representación'; estos nombres se emplean para designar impersonalmente al que o los que desempeñan la función o cargo: 'instrucciones de la dirección, la legación de España...'.

-ada: Sufijo con que se forman: a) Nombres de acción derivados de verbos o nombres: 'llamada, cornada'.

-dura: Parte invariable de los sufijos «-adura, edura, -idura», con que se forman nombres verbales de acción, efecto, utensilio, residuos, etc.: 'selladura, metedura, partidura; cerradura; raspaduras, barreduras; colgadura, añadidura». También, nombres derivados de otro, a través de un supuesto verbo: 'botonadura'. (V. «-ura»).

-miento (-imiento): Sufijo con el que, lo mismo que con «-mento», se forman nombres de estado, acción o efecto: 'atrevimiento, acaloramiento, sostenimiento’. (V. observación en «-ción»).

En el Diccionario General de la Lengua Española (DGLE) se incluyen tan sólo los sufijos actualmente más productivos.

(3) Definiciones del DGLE

-ción: 1 sufijo que entra en la formación de nombres que significan acción y efecto, gralte. derivados de verbos. La mayoría son de origen latino: abdicación, abolición; los de origen español se derivan de verbos, gralte. de la $1^{\mathrm{a}}$ y $3^{\mathrm{a}}$ conjugación, y toman respectivamente las formas -ación (alteración) o -ición (fundición). Existen algunos derivados de nombres, tomados éstos, gralte., de su forma latina: aera- 
ción, aviación.

-miento: 1 sufijo que entra en la formación de palabras con el significado de efecto o acción: fingimiento, alumbramiento; toma la forma amiento en los derivados de verbos de la $1^{\text {a }}$ conjugación; -imiento en los de la $2^{\mathrm{a}}$ y $3^{\mathrm{a}}$; y -mento en los latinos cultos: fundamento, impedimento.

Podemos ver que la información que se da de los sufijos en este diccionario es igual a la que aportan los otros dos diccionarios ya vistos: se señala la forma del sufijo y sus variantes y el significado del mismo con la etiqueta de "acción y/o efecto".

En contraste, en el diccionario CLAVE los sufijos no aparecen en el cuerpo de la obra (a diferencia de los prefijos) sino que se registran en un lista final (anexo IV). Esta presentación de los sufijos, según establecen los criterios de elaboración del diccionario, "tiene el objetivo de enriquecer la competencia lingüística de los hablantes, a fin de hacerlos capaces de "deducir" el significado de palabras nuevas a partir del significado de los elementos que los componen”. Así, y en orden alfabético, registran los distintos sufijos, indicando la categoría gramatical de la palabra resultante, el significado del mismo y algún ejemplo:

(4) Definiciones de CLAVE

\begin{tabular}{|c|c|c|}
\hline ión & s. Acción y efecto & acusación, demolición \\
\hline$-d a$ & s. Acción y efecto & llegada, cogida, salida \\
\hline 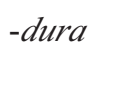 & $\begin{array}{l}\text { s. Acción y efecto } \\
\text { Instrumento }\end{array}$ & $\begin{array}{l}\text { raspadura, rozadura } \\
\text { cerradura }\end{array}$ \\
\hline & s. Acción y efecto & cargamento, impedi \\
\hline & s. Acción y efecto & alejamiento, cocin \\
\hline
\end{tabular}

En cambio, en ningún sitio se señala la categoría de la base a la que el sufijo se adjunta, sino que esta debe extraerse de los ejemplos. Este tipo de representación presenta, además de los mismos problemas que hemos señalado en los otros diccionarios para la definición del significado de los sufijos, un problema añadido y es que el criterio de elaboración establecido no justifica el distinto tratamiento que reciben los afi- 
jos. Si se decide incorporar en la macroestructura del diccionario los afijos de una lengua creemos que deben incorporarse todos y no sólo una parte, es decir, no se fundamenta que los prefijos sí reciban esa atención y los sufijos no. Esta misma consideración debemos extenderla al Diccionario del español actual que no registra en ningún lugar los sufijos aunque sí incluya los prefijos en el cuerpo de la obra.

Lo visto hasta el momento muestra que los sufijos que forman nombres derivados de acción no reciben una representación lexicográfica consistente en los diccionarios actuales. El motivo principal creemos que es la dificultad de explicar con precisión (y preferentemente en poco espacio) las características inherentes de cada sufijo, es decir, determinar no sólo la base a la que se adjunta y las variaciones morfofonológicas que presente, sino también las modificaciones semánticas, si las hubiera, que provoca el sufijo. Y atendiendo concretamente a este último aspecto, la confusión de asignar a los sufijos no sólo el contenido asociado a la nominalización, que es el de 'acción' o 'evento' marcado por la base de derivación, sino también el atribuirle el contenido de las distintas desviaciones o extensiones que presenten de forma particular las palabras derivadas.

Hasta ahora hemos visto cómo son definidos los sufijos nominalizadores en los diccionarios del español actual; veamos a continuación como se describen los sustantivos derivados.

\section{LOS NOMBRES DE ACCIÓN EN LOS DICCIONARIOS DEL ESPAÑOL}

Los diccionarios generalizan al máximo posible la paráfrasis definitoria de estos nombres deverbales, hecho que provoca graves desajustes en la información que transmiten al usuario.

Con la excepción del diccionario CLAVE, los diccionarios recurren sistemáticamente a la fórmula de 'acción y/o efecto' del verbo determinado. Esta definición tan concisa y abstracta ha suscitado dudas y aun discusiones entre los lexicógrafos. Ya en 1918 el director de la Academia, consciente de la inexactitud e irregularidad en la aplicación de la 
etiqueta, promovió un estudio (Ribera 1918) sobre el sentido exacto de la palabra efecto ${ }^{3}$. A pesar de todo esto, la fórmula se ha ido transmitiendo y perdurando en la tradición lexicográfica hasta llegar a nuestro días.

Si la analizamos, la definición puede ofrecer a primera vista algunas hipotéticas ventajas como es el hecho de eludir la repetición de la definición del verbo, lo que permite una mayor economía de espacio, o indicar con claridad la etimología de la palabra al señalar el verbo del que deriva el nombre de acción. Cuestiones, no obstante, que tienen su expresión adecuada por otros medios (abreviaturas para la expresión gramatical y cursiva para la etimología, por ejemplo). Pero lo que sí tiene es una desventaja grave: deja inexplicado el sentido de la palabra.

Partamos de una situación cotidiana. Si buscamos una palabra tan conocida como composición en el Diccionario del español actual $(D E A)$ nos encontramos en su definición como primera acepción: 'Acción de componer. También su efecto' y como una subacepción, es decir, con un sentido que, sin separarse marcadamente del presentado, se especializa en algún matiz, 'obra escrita artística, especialmente musical'. El significado de acción de esta unidad léxica parece claro al usuario, sin embargo, ¿qué debe entenderse por efecto de componer que no sea una obra, sea ésta de la naturaleza que sea? Si acudimos al diccionario de la Academia (1992) para resolver esta duda nos encontraremos con la siguiente definición:

(5) composición ${ }^{4}$. (Del lat. compositio, -onis). \|1 1. f. Acción y efecto de componer. || 2. Ajuste, convenio entre dos o más personas. || 3. Compostura, circunspección. || 4. Obra científica, literaria o musical. || 5. Poema, texto de sentido unitario, normalmente en verso. || 6. desus. Oración que el maestro de gramática dictaba en romance al discípulo para que la tradujera a la lengua que aprendía. || 7. Escrito en

3 Remitimos al citado artículo para observar principalmente como se propagó la palabra efecto desde el diccionario de Autoridades hasta los diccionarios actuales.

4 En la edición del 2001 la microestructura del artículo es básicamente la misma aunque se ha alterado el orden de algunas acepciones y se ha suprimido la que ya se señalaba en desuso. 
que el alumno desarrolla un tema, dado por el profesor o elegido libremente, para ejercitar su dominio del idioma, su habilidad expositiva, su sensibilidad literaria, etc. || 8. Ferr. Conjunto de los vagones que forman un tren. || 9. V. bula de composición. || 10. Der. Arreglo, generalmente con indemnización, que permitía el derecho antiguo sobre las consecuencias de un delito, entre el delincuente y la víctima o la familia de esta. || 11. Gram. Procedimiento por el cual se forman vocablos agregando a uno simple una o más preposiciones o partículas u otro vocablo íntegro o modificado por eufonía; v. gr.: anteponer, reconvenir, hincapié, cejijunto. || 12. Impr. Conjunto de líneas, galeradas y páginas, antes de la imposición. || 13. Mús. Parte de la música que enseña las reglas para la formación del canto y del acompañamiento. \| 14. Esc. y Pint. Arte de agrupar las figuras y accesorios para conseguir el mejor efecto, según lo que se haya de representar.

A pesar de la pretendida exhaustividad con la que se nos define el sustantivo, la interpretación de efecto sigue en la más absoluta oscuridad. Es más, la utilización de la fórmula tan concisa en la primera acepción de la palabra parece carecer de sentido cuando se recurre a procedimientos que podríamos calificar de antieconómicos para la definición de la palabra.

Los diccionarios nos proporcionan, pues, informaciones muy insuficientes con la utilización arbitraria de la citada etiqueta. Además presentan una desventaja más que podríamos calificar como metodológica. Esto es, se vulnera una de las normas iniciales que indican los diccionarios en sus respectivos preámbulos: las distintas acepciones de una unidad léxica deben ir aparte dentro del mismo artículo lexicográfico. Con esta fórmula se han juntado, sin embargo, las nociones de acción y efecto como si formasen una sola cuando en realidad constituyen dos acepciones distintas, como lo son también las otras extensiones semánticas que particularmente puede desarrollar el sustantivo.

Veamos el caso de dos nombres deverbales como son construcción y salida. Ambos poseen el significado primigenio de 'evento' marcado por el verbo base de derivación. Sin embargo, a partir de este valor inicial han desarrollado unas extensiones semánticas diferentes, condicionadas, sin duda, por las propiedades del verbo que contienen. Por ejemplo, un sustantivo como construcción se interpreta como la acción de construir en la 
secuencia (6a) y como el efecto o resultado en la secuencia (6b):

(6) a. La construcción de la autovía se retrasará hasta finales de año

b. La construcción será inaugurada por el presidente de la diputación provincial

De igual forma que el sustantivo salida posee la interpretación de acción y la de lugar, como vemos en (7):

(7) a. El tren de Alta Velocidad efectuará su salida en breves momentos

b. La salida está indicada con letreros luminosos

Y carece de la acepción de resultado o efecto, por más que nos lo señale el diccionario académico o el $D G L E$, como vemos:

(8) salida:

DRAE f. Acción y efecto de salir o salirse. \| 2. Parte por donde se sale fuera de un sitio o lugar.

DGLE 1 Sustantivo femenino. Acción de salir. 2 Efecto de salir. 3 Acción de salir un astro, cuerpo celeste. 4 Instante en que se produce dicha acción. 5 Parte por donde se sale fuera de un lugar.

Es más, ¿qué se entiende de nuevo por 'efecto de salir'? La paráfrasis utilizada plantea al usuario una nueva duda sobre el sentido exacto de la palabra objeto de su búsqueda.

Sin duda, la formula única es lo más cómodo para el que redacta el diccionario, pero no lo es para los lectores. Los diccionarios nos proporcionan informaciones muy defectuosas que nos sirven sólo si las empleamos para descifrar mensajes ya emitidos, pero no si deseamos utilizarlas para generar nuevas secuencias.

A continuación, vamos a fijarnos más detenidamente en ciertas anomalías que surgen de un estudio más exhaustivo de la citada fórmula.

5 En la nueva edición del DRAE salida se nos define por remisión a salido/a. En la acepción cuarta y quinta de este lema encontramos los dos sentidos que se le asignan al nombre deverbal.

6 Excepto en $C L A V E$, que no recurre a esta paráfrasis definitoria al primar la explica- 
Desglosados sus componentes, nos ocuparemos en primer lugar de la utilización de la etiqueta 'acción' en los distintos diccionarios consultados ${ }^{6}$.

\subsection{LA ACEPCIÓN EVENTIVA DE LA NOMINALIZACIÓN}

La utilización de la palabra acción presenta ciertas anomalías para la definición de estos sustantivos. En esta sección comentaremos dos de los problemas que creemos más relevantes. En primer lugar, nos ocuparemos de las restricciones en la formación de nombres derivados que puede experimentar el verbo. En segundo lugar, de la incoherencia en la aplicación de dicha etiqueta para las nominalizaciones formadas sobre verbos estativos. Atendamos al primer caso.

Como venimos observando, los sustantivos deverbales poseen un significado inicial y primigenio que en los diccionarios se define como 'acción' del verbo correspondiente. Sin embargo, los verbos desarrollan muy comúnmente acepciones contextuales, generalmente metafóricas, en los que no es posible la nominalización y que en ningún caso se señala en los diccionarios que consultamos. Veamos algunos ejemplos ya señalados por Lázaro Carreter (1971).

El verbo casar además de su acepción de 'contraer matrimonio', posee otro sentido, evidentemente metafórico, que los diccionarios califican de figurado, el de 'hacer coincidir o armonizar'. La nominalización del verbo correspondiente es sólo posible en la primera acepción del verbo:

(9) casarse una mujer con un hombre - el casamiento de una mujer con un hombre

Pero no tiene correspondencia en la acepción contextual de casar las piezas de un puzzle (?el casamiento de todas piezas del puzzle es muy dificultoso), por ejemplo. No obstante, la información que nos ofrecen los diccionarios de la palabra con su definición de "acción de casar o casarse" no indica en ningún caso la imposibilidad de producir dicha

ción del significado de la palabra sobre la concisión y definición global válida para todos los nombres deverbales que permite la fórmula. 
secuencia.

En condiciones semejantes a las descritas, la lengua introduce en otras ocasiones otro vocablo de la misma familia etimológica para permitir la nominalización del verbo en determinadas acepciones contextuales. Así, el verbo romper tiene asociado los sustantivos de rompimiento, rotura y ruptura. Sin embargo, aunque los diccionarios los definen de forma análoga, no pueden utilizarse indistintamente. No decimos la rotura del acuerdo, sino la ruptura del acuerdo; como no decimos la ruptura de las costuras del pantalón sino la rotura de las costuras. De igual manera, los sustantivos aclaración y aclarado indican la acción de aclarar; sin embargo, el primer término se utilizará en un contexto como la aclaración de su respuesta, y el segundo en el aclarado de la camisa.

Veamos otro ejemplo. Los diccionarios nos informan de que recepción es la acción de recibir, al igual que recibo. Por lo que, en principio, cabría pensar que recibo y recepción son equivalentes, aunque para el uso actual del español el sustantivo recibo carece de la interpretación eventiva. De cualquier forma, no obstante, ni recepción ni recibo son posibles en un contexto como recibir una visita.

Parece obvio que un diccionario concebido con el propósito de dar cuenta de lo que ya sabemos sobre la estructura del léxico, debería precisar, en cada caso, y creemos que bajo la entrada del propio sustantivo, las restricciones en las nominalizaciones que experimenta el verbo. Si los sememas del verbo aparecen ordenados, la entrada de su correspondiente nominal deberá indicar a partir de cuál de ellos es posible la nominalización. Una cuestión que puede solucionarse por medio de una simple señal que remita a la acepción verbal correspondiente o bien recurrir al procedimiento adoptado por la RAE para algunas entradas en la nueva edición de su diccionario, como es el caso de casamiento:

(10) casamiento: $\mathrm{m}$. Acción y efecto de casar ( || contraer matrimonio) (DRAE-2001)

El lema casamiento se define como 'la acción y el efecto' del verbo casar en su acepción de 'contraer matrimonio'. Una solución, no obstante, poco económica, ya que repite la definición completa del verbo 
en la que es posible la nominalización.

Detengámonos a continuación en otro problema que observamos en la aplicación de la palabra acción para definir estos nombres.

La utilización de la etiqueta acción lleva también consigo otro tipo de incoherencias gramaticales. Una de las más apreciables es la descripción de los nombres formados sobre los denominados verbos estativos. Se trata de aquellos verbos que indican situaciones estables que tienen que ver con propiedades del sujeto, y no con acciones que puedan llevar a cabo, como la cualidad, su disposición, la habilidad, etc., como pueden ser conocer, ignorar, estar o habitar. Excepto en el CLAVE, en los diccionarios consultados se siguen describiendo generalmente como 'acción' del verbo correspondiente. Así en el Diccionario general de la lengua española y en el DRAE, el sustantivo conocimiento se describe como 'acción y efecto de conocer'. Una denotación incompatible con las características del propio verbo, que denota un estado psicológico en ningún caso controlado por el sujeto. Pueden darse también inconsecuencias al describir de un modo u otro estos nombres. Si para conocimiento el diccionario académico ha decidido recurrir a la reiterada fórmula, para el sustantivo ignorancia, también deverbal, decide darnos la siguiente información:

(11) ignorancia: Falta de ciencia, de letras y noticias, o general o particular.

En este caso se evita la fórmula. Creemos que con el propósito de explicar con mayor exactitud el sentido de esta palabra, aunque el carácter didáctico en la redacción de la definición pueda también discutirse.

Otros casos donde estos nombres son descritos con la etiqueta de 'acción y efecto' son: turbación, perturbación o enajenación, frente a desesperación o aburrimiento en los que se recurre a definiciones sinonímicas.

Atendamos ahora a dos casos paradójicos, como son la definición de los sustantivos habitación y estación en los diccionarios consultados. Resulta chocante que en un diccionario de uso encontremos las siguientes definiciones de habitación:

(12) habitación: 
DRAE: f. Acción y efecto de habitar. \|2. Edificio o parte de él que se destina a vivienda. || 3. Cualquiera de los aposentos de la casa o morada. || 4. Estrictamente, dormitorio.

DUE: 1. Acción de habitar. (T. «habitamiento, habitanza».) 2. En sentido amplio cualquier sitio en que habitan personas o animales.

$D G L E$ : 1 Sustantivo femenino. Edificio o parte de él que se destina a vivienda. 2 Pieza, esp. aquella en que se está o se duerme. 3 Acción de habitar. 4 Efecto de habitar.

En el caso de estación, estos diccionarios nos ofrecen como primera acepción de la palabra 'estado actual de una cosa'. El diccionario académico en su edición de 1992 especifica que se trata de un significado en desuso; en su vigésima segunda edición esta acepción ha sido ya suprimida.

A partir de estos dos ejemplos, podemos observar una descripción incoherente con el empleo de la etiqueta de 'acción' de dos sustantivos deverbales de idéntico comportamiento. El nombre deverbal estación se ha definido, no obstante, con mayor exactitud al recurrir al vocablo 'estado' y no al de 'acción'.

Pese a ello, este tipo de definiciones nos está desvelando, por otro lado, el procedimiento morfológico de la formación de estos nombres, tal y como lo describimos en las páginas iniciales. Es decir, cómo el proceso de formación de nombres deverbales en español dota al sustantivo derivado de un significado eventivo inicial ('acción'o 'estado') que está determinado por la naturaleza semántica del verbo base. Una vez incorporado al léxico y en virtud de un desplazamiento metonímico, el sustantivo desarrolla otras significaciones determinadas por la propia base de derivación y según requieran las necesidades comunicativas y lingüísticas de los hablantes. En el caso de las definiciones de habitación y de estación, se trata, sin duda, de las acepciones primigenias que poseían estas palabras pero que están fuera de la memoria de los hablantes actuales y, por tanto, deberían estar excluidas de un diccionario de uso del español actual.

Otro problema que cabría añadir a los ya citados es el hecho de que otros sustantivos también considerados deverbales desde la sincro- 
nía, único factor determinante en un diccionario de uso, no reciben el mismo tratamiento, es decir, no se definen con la fórmula global de 'acción de V'. La razón de esto, generalmente, por atender a criterios etimológicos. Así, el sustantivo diseño, de origen italiano, no se define con la citada etiqueta, sino que se recurre a una explicación sinonímica y se señala el étimo del que procede. En el caso de ruina, por ejemplo, por atender a un confuso sistema de definiciones y referencias cruzadas. Este sistema basa la definición del vocablo en la determinación de su carácter de primitivo o derivado. Así, la palabra primitiva llevará una explicación extensa. La derivada se definirá por referencia a su primitivo. En ruina, por ejemplo, se recurre a una explicación extensa por ser considerada como la forma primitiva de la que deriva el verbo arruinar y se define como 'acción de destruir(se)'. Este sustantivo no se califica, por consiguiente, como la 'acción' del verbo arruinar(se), sino del verbo destruir(se), porque, al suponerse como primitivo, no puede basar su definición en la remisión de un verbo que se deriva de él. Es el verbo el que se define por remisión al sustantivo. Así encontramos arruinarse: 'causar ruina', haciendo referencia a la definición dada en ruina. Se trata de un sistema muy controvertido por la dificultad de fijar con exactitud en muchas ocasiones cuál es la palabra primitiva y que, en cualquier caso, hace referencia a la etimología del vocablo. Una cuestión que tiene su expresión adecuada por otros medios y no debe incluirse en la explicación del sentido del mismo.

En otros casos, el hecho de que los sustantivos derivados no reciban este tratamiento se debe a descuidos o porque simplemente no habrá llegado el momento de aplicar la conocida etiqueta a casos como masturbación, dosificación, venganza, abnegación, vigilancia, etc. en el diccionario académico, por ejemplo.

Vamos a ocuparnos ahora de la acepción de 'efecto' con la que sistemáticamente se definen también estos nombres.

\subsection{LA ACEPCIÓN RESULTATIVA DE LA NOMINALIZACIÓN}

Si la aplicación de la etiqueta de acción para definir estos sustanti- 
vos derivados presentaba ciertas anomalías, no menos inexactitud encontramos en la utilización de la palabra efecto. La causa principal de esto, como veremos, es su carácter excesivamente genérico para que pueda servir de explicación concreta y clara del sentido de una palabra. Veámoslo.

Partamos del supuesto de que con efecto queremos denotar el resultado producido por una determinada acción, por lo que habrá que determinar y especificar las características del predicado verbal que nos permitan definir su resultado, si lo hubiera, con exactitud.

Extraemos de cuatro diccionarios (DRAE, DGLE, DUE y DEA) las siguientes entradas de nombres deverbales, consignando únicamente sus principales acepciones:

(13) construcción

$D R A E$ f. Acción y efecto de construir. || 2. [f.] Arte de construir. || 3. [f.] Tratándose de edificios, obra construida.

DGLE 1. Acción de construir. 2 Efecto de construir. 3 Tratándose de edificios, obra construida. 4 Arte de construir.

DUE Acción y efecto de * construir. Arte y actividad de construir edificios. Cosa *construida. Particularmente, *edificio, fábrica u obra de albañilería

DEA 1. Acción de construir. 2. Actividad de construir edificios. 3. Cosa construida.

(14) producción

DRAE f. Acción de producir. || 2. Cosa producida. || 3. Acto o modo de producirse.

DGLE 1. Acción de producir. 2 Cosa producida, producto. 3 Acto o modo de producirse.

DUE Acción de producir. «Producto». Cosa producida.

DEA 1. Acción de producir. 2. Cosa o conjunto de cosas producidas.

(15) interpretación

$D R A E$ Acción y efecto de interpretar.

DGLE 1 Acción de interpretar. 2 Efecto de interpretar. 
DUE Acción y efecto de interpretar.

$D E A$ Acción y efecto de interpretar.

(16) clasificación

$D R A E$ Acción y efecto de clasificar.

$D G L E \quad 1$ Acción de clasificar. 2 Efecto de clasificar.

DUE Acción y efecto de clasificar.

DEA 1. Acción de clasificar(se). 2. Lista en que están ordenados por clases los elementos de un subconjunto.

En los diccionarios que hemos consultado podemos ver cómo bajo la etiqueta de efecto se están designando entidades muy diferentes. Nuestra propia intuición de hablantes aprecia que los nombres deverbales producción y clasificación en su denotación de efecto son distintos resultados del proceso verbal, como vemos en (17):

(17) a. La producción de este año ha sido muy superior a la del año anterior

b. No me gusta nada la clasificación de los libros que ha hecho Luis

La teoría así lo corrobora. Las dos primeras entradas corresponden a nominalizaciones sobre verbos denominados de objeto resultado o efectuado. Las siguientes a las de los denominados verbos de objeto afectado. La clase verbal de construir o producir (verbos de objeto efectuado) se caracteriza por comprender a predicados cuya acción va dirigida a objetos que no existen previamente a la acción, sino que éste es creado, efectuado, por la misma. Por lo tanto, el resultado de dicha acción designará un objeto nuevo. Valor que metonímicamente ha adquirido el sustantivo deverbal producción, como se aprecia en (17a). Por su parte, la acción indicada por los verbos interpretar y clasificar (verbos de objeto afectado) va dirigida a objetos ya existentes que sufren una modificación. El sustantivo en su valor de resultado designará no un objeto nuevo producido por la acción verbal sino un cambio de estado del objeto ya existente, como vemos en el deverbal de (17b).

El valor tan genérico del término efecto no aclara en ningún caso el sentido de la palabra. Es más en muchos casos no sólo la deja inexplica- 
da sino que provoca nuevas dudas al usuario, como vemos en la entrada de construcción, indicada en (13). En los diccionarios consultados se nos informa que construcción puede indicar la acción y el efecto del verbo construir y además, como si de una acepción nueva se tratase, se indica 'obra construida' cuando en realidad se trata del mismo efecto, lo que el usuario puede comprobar consultando simplemente la entrada del verbo construir. Este tipo de incongruencia es debida, sin duda alguna, a la reiteración mecánica de la fórmula de 'acción y efecto'.

Por lo tanto, la causa de la inexactitud en la definición de estos sustantivos deverbales se encuentra tanto en el carácter excesivamente genérico de la etiqueta de 'efecto' como en el uso reiterado y arbitrario que se hace de la misma, que lleva a casos como el de construcción; en otros a indicar efectos donde no los hay, como el caso de salida, flotación, reedificación e, incluso, en natación; o en otros, en cambio, a no señalar el efecto aunque éste se pueda concebir fácilmente, como en el caso de apelación o predicación.

\section{CONCLUSIÓN}

En lo que nos ha sido posible, hemos señalado los problemas que posee la representación lexicográfica de los denominados nombres deverbales de acción en los diccionarios de uso del español. En primer lugar, hemos mostrado que la descripción que ofrecen de los sufijos no es adecuada. Se asigna a los sufijos no sólo el contenido asociado a la nominalización, que es el del evento (generalmente, acción) marcado por el verbo al que se adjuntan, sino que además se les atribuye otros valores semánticos (efecto, instrumento, lugar, etc.) de los que en realidad carece, ya que son el contenido de las distintas extensiones o desviaciones de significado que presentan de forma particular los sustantivos derivados.

A continuación hemos presentado los inconvenientes que presenta la paráfrasis con la que sistemáticamente se definen estos nombres en los diccionarios del español. Los artículos destinados a estos sustantivos derivados son descritos con la etiqueta de 'acción y efecto' del verbo correspondiente sin tener en cuenta que con dicha fórmula se han juntado dos acepciones distintas de una misma palabra. Al mismo tiempo se 
generan graves desajustes en la información que transmiten al usuario. En primer lugar, vimos que se generaliza la posibilidad de la formación de la nominalización en todas las acepciones del verbo. Vimos además que se utiliza la etiqueta de 'acción' para nombres formados sobre verbos estativos y, a continuación, que la recurrida palabra efecto es demasiado genérica para que pueda servir de explicación concreta y clara. En suma, esta definición tan concisa de las nominalizaciones ha venido a ser oscura y casi ininteligible y su utilización mecánica y rutinaria.

La mejora de la descripción lexicográfica de estos nombres implicará en un principio:

- Separar las distintas acepciones que poseen estos sustantivos. Como paso inicial, las que se incluyen en la repetida fórmula: 'acción' y 'efecto'. Una propuesta ya sugerida por Ribera (1918) y que parece que no ha tenido la repercusión necesaria. Acometer dicho trabajo significa afrontar la arbitrariedad con que se suele tratar el problema de la polisemia en los nombres deverbales y la falta de distinción entre el significado general de los sustantivos creados por este proceso morfológico y los significados periféricos que puedan desarrollar. Por ejemplo, el sustantivo construcción tendrá dos acepciones distintas y separadas. Una primera acepción que será la del 'evento' del verbo correspondiente. Una segunda, la del resultado de dicha acción.

- Escoger y utilizar el término más adecuado que defina el significado primigenio que poseen estos nombres. Proponemos o bien el más general de 'evento' o bien, por continuar con la tradición lexicográfica, el de 'acción' y el de 'estado', según convenga a las características semánticas del verbo del que proceden ${ }^{7}$. Así dos sustantivos como construcción y conocimiento podrían definirse con la misma etiqueta de 'evento' indicado por el verbo base. En la entrada del verbo se especificarán las características de dicho

7 Por tanto, la etiqueta evento englobará la denominación tradicional de 'acción' y la más específica de 'estado'. 'Acción’ y ‘estado' recogerán, así, la distinción aspectual de los verbos a los que se adjuntan los sufijos nominalizadores. 
evento. O bien se podría optar por especificarlas en la entrada de la propia nominalización. En ese caso, construcción se definiría como 'acción' de construir. En el caso de conocimiento de 'estado' del verbo correspondiente.

- Remitir a la entrada verbal correspondiente y a la acepción del verbo en la que es posible la nominalización. Por ejemplo, el deverbal aclaración se definirá como la 'acción' o ‘evento' de aclarar en su primera acepción.

- Adecuar esta definición a las distintas entradas con términos más específicos si la explicación de la palabra no se deduce con facilidad porque el usuario no vea de forma clara la relación formal y semántica del nombre con el verbo y, por consiguiente, no alcance a comprender el significado del mismo, o bien porque su utilización está circunscrita a unos contextos determinados, como pueden ser los casos de los nombres derivados de abstenerse: abstención y abstinencia.

Mediante estas pautas se conseguirá una mejor acomodación a las instrucciones que figuran en el frente de los diccionarios para definir con claridad las palabras de una lengua pero, sobre todo, servirá para revisar de forma detenida los artículos destinados a estos nombres y rectificar algunos errores que inevitablemente se deslizan a partir de la utilización de la etiqueta y que hemos visto al analizar el modo en que son definidos. Al mismo tiempo, se corregirá también la inconsecuencia de que multitud de nombres deverbales no tengan este calificativo, en unos casos porque se atiende a la etimología (caso de diseño) o la dirección de la derivación (caso de ruina), en otros porque simplemente no habrá llegado el momento de aplicársela, como los casos ya señalados de masturbación, dosificación, venganza, abnegación, vigilancia, etc. en el diccionario académico.

La calidad en la descripción lexicográfica de estos sustantivos se incrementará cuando los diccionarios aporten información sobre la subcategorización de los verbos, base de derivación de este proceso derivativo y, por extensión, la de los propios nombres derivados, siempre, claro está, de forma que pueda ser fácilmente interpretada por un usuario me- 
dio. 